\title{
Anabases
}

ANABASES Traditions et réceptions de l'Antiquité

$10 \mid 2009$

Varia

\section{Historien de l'Égypte grecque et romaine : trois parcours intellectuels croisés}

\section{Bernard Legras}

\section{(2) OpenEdition}

1 Journals

Édition électronique

URL : http://journals.openedition.org/anabases/633

DOI : 10.4000/anabases.633

ISSN : 2256-9421

Éditeur

E.R.A.S.M.E.

\section{Édition imprimée}

Date de publication : 1 octobre 2009

Pagination : 75-88

ISSN : 1774-4296

\section{Référence électronique}

Bernard Legras, «Historien de l'Égypte grecque et romaine : trois parcours intellectuels croisés »,

Anabases [En ligne], 10 | 2009, mis en ligne le 01 octobre 2012, consulté le 14 novembre 2019. URL

http://journals.openedition.org/anabases/633 ; DOI : 10.4000/anabases.633 
Anabases 10 (2009), p. 75-88.

\title{
Historien de l'Égypte grecque et romaine: trois parcours intellectuels croisés
}

BERNARD Legras

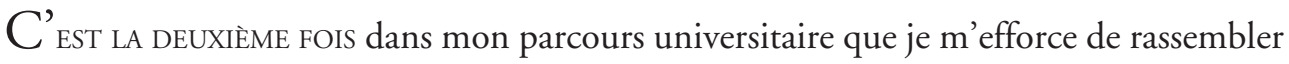
des éléments permettant de reconstituer mon parcours intellectuel et d'en déterminer les filiations qui fondent le désir d'écrire et de transmettre. Le premier essai faisait partie des règles qui s'imposent en France depuis 1984 à tout enseignant-chercheur aspirant à l'habilitation à diriger des recherches, et donc au droit d'encadrer et de former des doctorants, c'est-à-dire les chercheurs qui perpétuent la chaîne du savoir innovant. Cette ego-histoire eut peu de lecteurs : l'expert chargé à l'université Paris 1 PanthéonSorbonne de déclarer recevable un dossier d'HDR (le romaniste Jean-Michel David), les membres de mon jury constitué autour de Jean-Marie Bertrand garant de mon HDR, Alain Blanchard, Michel Chauveau, Geneviève Husson et Joseph Mélèze Modrzejewski, puis quelques amis désireux d'avoir sous les yeux un exemple pour l'écriture de leur propre manuscrit. L'ambitieux projet initié par la revue Anabases, qui impose de croiser plusieurs réflexions, me conduit à publier ici un court passage de ce texte de 81 pages écrit durant l'été 2003, pour dresser un nouveau bilan, six ans plus tard. Au terme d'un parcours de plus de trois décades - alors que nombre de mes maîtres sont encore actifs je me suis efforcé d'expliquer tant les chemins empruntés que ceux que j'ai pu délaisser, ne serait-ce qu'un temps, dans des recherches toujours inachevées.
\end{abstract}




\section{2003, l'ultime rite de passage universitaire: l'écriture d'une ego-histoire}

\section{Introduction : extraits}

"Cette "ego-histoire" trouve son origine, un mercredi après-midi de l'automne 1977, en Sorbonne, en salle Picard, quand le professeur Joseph Mélèze Modrzejewski commença son cours d'initiation à la papyrologie documentaire. Les charmes de la discipline se révélèrent vite à l'étudiant en Licence d'histoire [au sein de l'université Paris 1 Panthéon-Sorbonne] que j'étais alors. Je découvris, au sein d'un petit groupe d'étudiants, des textes qui font pénétrer dans la vie des habitants de l'Égypte hellénistique et romaine sous tous ses aspects. L'étude du contrat de mariage d'Éléphantine, d'un prostagma ptolémaïque, ou d'une déclaration de recensement romain, me firent rapidement comprendre la diversité de ces sources, dont je pouvais apprécier la spécificité grâce aux autres cours suivis dans le cadre d'un précieux enseignement aux "Sciences auxiliaires de l'histoire grecque". Je découvrais en effet parallèlement la mycénologie avec le professeur Henri van Effenterre, et l'épigraphie avec monsieur François Aron, maître de conférences enthousiaste et exigeant, qui accompagnèrent avec attention, l'un et l'autre, mon parcours d'étudiant puis de jeune chercheur.

Cette année fut décisive, puisqu'elle m’orienta tout naturellement vers une maîtrise d'histoire ancienne fondée essentiellement sur les sources papyrologiques. Ce choix avait certes été préparé par d'autres faits ou événements liés à ma paideia. Naître fils d'un agrégé de grammaire contribua à me faire découvrir dès le collège les joies (et les labeurs) de l'étude des lettres anciennes: latin en sixième (année scolaire 1967/1968) et grec en quatrième. L'allemand s'imposait comme première langue dans une famille venue d'Alsace et de Lorraine. Cette scolarité au Lycée JacquesDecour (Paris IX') m'offrit ainsi le socle indispensable pour accéder à l'Altertumswissenschaft. L'obtention d'un baccalauréat scientifique allait quelque peu contre mes goûts qui s'étaient tournés vers l'histoire durant la classe de Première, grâce à l'enseignement d'un maître exceptionnel, Pierre Raison. Je passai sans succès les épreuves du concours général d'histoire, mais cette préparation me permit de mettre à l'épreuve cette jeune vocation.

Une hypokhâgne et deux khâgnes, à Henri IV, la renforcèrent, grâce à l'enseignement de ces grands préparateurs qu'étaient, pour l'histoire, le romaniste Marcel Bordet et le contemporanéiste Max Tacel, Maurice de Balmann pour le grec. Les hasards des programmes de l'ENS Ulm firent que "Les Lagides" me devinrent alors familiers. Le cours préparé par Max Tacel était un résumé d'Auguste Bouché-Leclercq. Je dois avouer que mes souvenirs sont surtout marqués par la complexité d'une question pour laquelle aucun manuel ne permettait de compléter le cours magistral avec un ouvrage récent. Les monographies d'Edwyn Bevan (Histoire des Lagides, 323-30 av. J.-C.) et de Pierre Jouguet (Égypte ptolémä̈que, dans Histoire de la nation égyptienne, G. Hanoteaux éd., 
t. III) dataient respectivement de 1934 (parution de la traduction française) et de 19331936. Ils n'étaient consultables qu'à la Bibliothèque Sainte-Geneviève, providence du khâgneux. Le lumineux manuel écrit par Claire Préaux dans la "Nouvelle Clio" ne serait publié qu'en 1978, mais j'ai appartenu de ce fait, avant de rejoindre la Sorbonne, à la "génération Bouché-Leclercq", avant d'entrer - sans transition - dans la "génération Mélèze Modrzejewski”.

Admissible à l'ENs Ulm, devenu Ipésien, je renonçai à faire une troisième khâgne, pour suivre les cours de l'université Paris 1 , où j'étais inscrit depuis mon entrée en hypokhâgne (1974). Mon service national accompli dans les Forces Françaises en Allemagne à Freiburg im Breisgau, je préparai ma maîtrise puis les concours de recrutement. Je réussis l'agrégation [d'histoire] dès la première année, $22^{\mathrm{e}}$ ex æquo sur un total de 54 reçus. Après l'année de stage [au Lycée G. Clemenceau de Villemomble, en Seine-SaintDenis], j’eus la chance d'être nommé au Collège Sévigné (Paris $\left.V^{\mathrm{e}}\right)$, établissement laïc privé sous contrat d'association avec l'État.

Je déposai sans tarder un sujet de thèse, sous la direction du professeur Joseph Mélèze Modrzejewski. Mais la soutenance fut retardée par les responsabilités administratives que j'assumai durant quatre ans comme proviseur du Collège Sévigné. Comprenant que cette fonction était incompatible avec la préparation d'un doctorat, je démissionnai, pour obtenir un poste d'enseignant au Lycée François-Villon (Paris $\mathrm{XIV}^{\mathrm{e}}$ ). Une charge de cours assurée à Paris 1 me montrait parallèlement tout l'intérêt de l'enseignement dans le supérieur. Madame le professeur Françoise Ruzé, qui m’avait parrainé dans cette entrée "par la petite porte" à Paris 1, avec le professeur Joseph Mélèze Modrzejewski, ne cessera durant ces années de m'entourer de ses conseils et de son amitié. Et l'ombre tutélaire du professeur Jean-Marie Bertrand, autre gardien du temple dédié à l'histoire ancienne qu'est le Centre Glotz (“Glotz"), n'était jamais très loin!

Je complétai ma formation en assistant à différents séminaires, principalement ceux du professeur Joseph Mélèze Modrzejewski (Papyrologie juridique et histoire des droits de l'Antiquité, EPHE, IV e section), de madame le professeur Claude Mossé (Histoire grecque, université Paris 7) et du professeur Alain Blanchard (Papyrologie littéraire, Institut de papyrologie de la Sorbonne, université Paris 4).

Outre ceux que je viens de préciser, deux éléments importants jouèrent un rôle dans ma vocation d'helléniste tourné vers l'Égypte grecque et romaine, d'une part des voyages de lycéen puis d'étudiant (sac au dos) qui me permirent de découvrir la Grèce et l'Asie mineure à une époque aujourd'hui révolue (premier voyage en Grèce à l'été 1973) et de découvrir in situ le cadre (paysages, peuple, traditions) de la civilisation grecque, d'autre part, une sensibilité pour les régions frontières, les carrefours, ainsi que les contacts et les échanges culturels.

Cette activité scientifique s'est déroulée dans le cadre des recherches en bibliothèque, en premier lieu à l'Institut de papyrologie de la Sorbonne, à la Bibliothèque nationale de France, au Centre Gustave-Glotz et à la Bibliothèque de la Sorbonne, mais aussi dans le cadre des congrès internationaux. Pour les conditions de travail en bibliothèque, 
la grande date est indéniablement la naissance d'un nouveau site de la $\mathrm{BnF}$ en bord de Seine à Tolbiac. Si j'ai fait partie dans les six premiers mois de l'ouverture du site "François-Mitterrand" du groupe des nostalgiques de Richelieu, qui burent, le dernier jour d'ouverture de la salle Labrouste, le champagne dans la salle de lecture, j'ai ensuite été séduit par la salle Rez-de-Jardin, ses livres en libre accès, ses collections enrichies et les contacts scientifiques et amicaux rendus possibles par la présence de collègues de plus en plus nombreux. L'informatisation de l'Institut de papyrologie de la Sorbonne a également été un moment important, qui exprime la vitalité d'une discipline pionnière en ce domaine, de même que le sera dans un très proche avenir la constitution rue Vivienne de la Bibliothèque "Gernet-Glotz" où travaillera mon équipe de recherche, Phéacie, désormais hébergée avec le Centre Gustave-Glotz et le centre Louis-Gernet dans les locaux de l'Institut National d'Histoire de l'Art. Les liens papyrologiques internationaux en particulier avec les collègues belges, seconde patrie de tout papyrologue en raison du rayonnement des centres d'importance mondiale que sont Bruxelles, Liège et Louvain (Leuven), sont partie prenante de ma formation. Je terminerai cette courte biographie scientifique par la mention de l'importance décisive qu'a revêtue pour moi la création de l'équipe de recherche aujourd'hui équipe d'accueil (EA 3563) "Phéacie: les pratiques culturelles dans les sociétés culturelles grecques et romaines" sous l'impulsion à Paris 1 de Pauline Schmitt Pantel et Jean-Marie Bertrand, et à Paris 7 de Louise Bruit Zaidman et Jean-Pierre Vallat. Cette équipe est à la fois un havre et un espace d'expression stimulant, qui me permet d'inscrire ma propre recherche dans un cadre inspiré entre autres par Louis Gernet, dont l'œuvre tournée vers le droit, l'anthropologie et la culture reste une référence fondamentale.»

Cet extrait peut être complété par le début de la présentation orale que je fis de mon dossier d'habilitation à diriger des recherches, le 20 novembre 2003 dans l'amphithéatre Louis-Liard, en Sorbonne.

"S’il ne fallait invoquer qu'un seul nom en dédicace de cette défense de soutenance, je ne prononcerais que le nom d'Ulrich Wilcken, qui publia magistralement en 1927 les papyrus grecs du Sarapieion, découverts au XIX ${ }^{\mathrm{e}}$ siècle sur le site de la nécropole de Memphis. L'œuvre de ce grand papyrologue, qui a été conduit devant le tribunal d'Osiris depuis plus d'un demi-siècle (il est mort en 1944) constitue aujourd'hui une somme inestimable, avec laquelle j'ai entretenu, depuis de nombreuses années, les rapports intellectuels les plus étroits, par la fréquentation quasi quotidienne de ses Urkunden der Ptolemäerzeit. Mais la perspective triangulaire qu'autorise - peut-être - la nature des travaux que j'ai l'honneur de présenter devant vous, puisque ma recherche s'est déployée - in fine - au pied des Pyramides de Saqqara, m'incite à citer également deux autres noms: en premier lieu celui d'Auguste Mariette, dont le square dédié au pacha égyptien fut créé en 1910 à quelques mètres de la Sorbonne, et en second lieu, Joseph Mélèze Modrzejeweski, sans lequel ma vocation d'historien du monde grec se serait peut-être dirigée vers d'autres horizons que l'Égypte ptolémaïque et romaine.» 


\section{2. À la recherche des origines: la vocation d'historien de l'Antiquité}

Les centres d'intérêt de ma recherche sont tournés vers trois domaines, le droit grec et hellénistique, la paideia grecque au double sens d'éducation et de culture, et les transferts culturels. S'il existe un lien entre les trois domaines, je le verrais volontiers dans la volonté d'étudier tout ce qui permet aux hommes de vivre ensemble, de dialoguer et d'échanger.

La conviction de l'unité des sciences historiques et la conscience des drames et des tragédies de l'histoire humaine ont alimenté un intérêt double pour l'Antiquité et le monde d'aujourd'hui. Je n'ai jamais connu la tentation qui saisira Johann Gustav Droysen et le détournera de l'histoire ancienne, une tentation qu'il exprime dans une lettre de 1841, écrite entre les deux volumes de l'Histoire de l'hellénisme parus respectivement en 1836 et en 1843: "Je suis fou de m'enterrer dans ce vieux passé poussiéreux au lieu de me jeter à corps perdu dans les époques vivantes, colorées, et plus proches de nous ${ }^{1}$.» Mes recherches me semblent plutôt marquées par cette «hantise du présent, de l'expérience vécue» que Claude Nicolet identifie dans l'écriture de l'Histoire romaine de Theodor Mommsen. L'enseignement des maîtres qui m'ont formé à la Sorbonne en était profondément empreint. Les cours et les séminaires de Joseph Mélèze Modrzejewski sur le judaïsme dans l'Égypte hellénistique et romaine, ont pour arrière-plan son vécu de rescapé de la Shoah, de chercheur quittant en 1958 la Pologne de Gomulka saisie par le démon antisémite ${ }^{2}$. Henri van Effenterre, dont j'ai reçu comme étudiant l'enseignement et que j'ai ensuite eu le bonheur de retrouver dans des colloques, en particulier lors des Symposia organisés par la Gesellschaft für griechische und hellenistische Rechtsgeschichte, était animé par une passion pour la pédagogie épousant les principes du scoutisme moderne, et un rapport quasiment charnel avec la Grèce contemporaine imprégnait cours et séminaires, à l'instar de ces lignes nostalgiques publiées en 1973: «La révolution commencée autrefois, grâce à de braves gens

1 Lettre à A. Heydemann du 27 février 1841 (Briefwechsel II, R. Hübner éd., Stuttgart, Berlin, 1929, p.442), citée par Benedetto Bravo, Philologie, histoire, philosophie de l'histoire. Etude sur J.G. Droysen historien de l'Antiquité, Wroclaw, Varsovie, Cracovie, 1968 (rééd. Hildesheim, Zurich, New York, Georg Olms, 1988), p.351-352, et Pascal PaYen, "Droysen, l'Histoire d'Alexandre le Grand et l'État prussien: la querelle de l'unité et la question du 'modernisme", dans Sylvie Caucanas, Rémy Cazals et Pascal Payen (éd.), Retrouver, imaginer, utiliser l'Antiquité, Toulouse, Privat, 2001, p. 129.

Biographie dans Dariusz Dlugosz (éd.), Grecs, Juifs, Polonais: à la recherche des racines de la civilisation européenne, Actes du Colloque international dédié à Joseph Mélèze Modrzejewski (Paris, 14 novembre 2003), Paris, Varsovie, Académie Polonaise des Sciences, 2006, p. 4-5 (et Witold Wolodkiewicz, p. 14-15). Cf. aussi Patricia Hidiroglou, "Pour le professeur Joseph Mélèze Modrzejewski", dans Entre héritage et devenir. La construction de la famille juive. Études offertes à Joseph Mélèze Modrzejewski, Paris, Publications de la Sorbonne, 2003, p.7-12. 
comme Spensithios, par l'introduction de l'alphabet phénicien, aboutit aujourd'hui à l'industrialisation de la Crète et à la mort de tout ce passé [...] Notre génération risque d'être la seule à pouvoir y retrouver le souvenir de Zeus, d'Hermès, d'Aphrodite et des Courètes, la dernière à y avoir connu des sanctuaires de sommet, des grottes sacrées et des bergers illettrés ${ }^{3}$.» Les "Rencontres Gustave-Glotz» organisées en Sorbonne, salle Picard, le lundi de $17 \mathrm{~h}$ à $19 \mathrm{~h}$, donnaient à Claude Nicolet l'occasion de développer des idées marquantes exprimées dans ces lignes: «Quand l'historien n'est pas un fonctionnaire besogneux, il ne connaît pas de sujets gratuits, ou contingents. L'histoire ancienne, où j'avais cru fuir une actualité trop pressante, n'était qu'un détour vers elle. Un détour nécessaire, d'ailleurs ${ }^{4}$.» Pierre Vidal-Naquet, qui appuiera plus tard, sans succès, mon désir de rejoindre l'Institut français d'archéologie orientale du Caire, fut de ces maîtres qui me marquèrent plus par la lecture que je faisais de leurs publications et par l'intérêt que j'éprouvais pour leur action publique d' "historien et de militant ${ }^{5}$ ". L'empreinte d'autres maitres se fit par la lecture de leurs ouvrages, ainsi pour JeanPierre Vernant ou Philippe Gauthier dont les séminaires me restèrent inaccessibles en raison de l'emploi du temps du professeur agrégé de lycée que j'étais durant la période d'écriture de ma thèse.

Cet intérêt pour le politique au présent m'a conduit - entre autres - à entrer dans le Comité de rédaction comme rédacteur-adjoint de la revue Crises publiée naguère par les Presses universitaires de France. Cette publication pluridisciplinaire, qui associait des politistes (Guy Hermet, Jean-Jacques Roche), des juristes (Michel Borgetto), des psychanalystes (Gérard Haddad, Jean-Jacques Moscowitz), des philosophes (Jean-Marc Lachaud, Thomas Stern), des écrivains (Jean-Pierre Faye, Bernard Lambert), des journalistes (Jean-Pierre Airut, Luce Perrot), des avocats (Serge Lewisch), des historiens (Alexandre Adler, Jean-Yves Camus, Annette Wieworka), s'interrogeait sur la notion de crises dans les sociétés humaines. L'aventure malheureusement écourtée par la décision de l'éditeur d'arrêter la revue au sixième numéro était belle. Le décloisonnement total des disciplines au sein du comité de rédaction, qui se réunissait chez le psychanalyste Ali Magoudi, autour du directeur de la revue, Yves Roucaute, professeur de sciences politiques, m'offrit de participer à des débats réunissant intellectuels de gauche et de droite, qui m'apportèrent des manières de penser différentes du monde universitaire traditionnel. J'ai toujours pensé que mon intérêt pour la notion de transferts culturels

3 Henri van Effenterre, "Le contrat de travail du scribe Spensithios", BCH 97 (1973), p. 31-46.

4 Claude Nicolet, La fabrique d'une nation. La France entre Rome et les Germains, Paris, Perrin, 2003, p. 14.

5 En lisant ses Mémoires je découvris avec émotion qu'il fit son stage pédagogique de trois semaines après sa réussite à l'agrégation au Lycée Jacques-Decour «avec M. Raison, membre d'une illustre tribu de professeurs" qui détermina précisément ma vocation d'historien en classe de première: Pierre VIDAL-NAQUeT, Mémoires, t. 2, le trouble et la lumière 1955-1998, Paris, Seuil/La Découverte, 1998, p. 13. 
qui s'éveillait alors, et pour l'étude des relations entre les hommes et les femmes a trouvé un vrai stimulus dans ce cercle ${ }^{6}$.

Le sixième numéro de Crises, que je préparais avec Claire Mouradian (directeur de recherches au CNRS) sur le génocide des Arméniens ne parut jamais. Mais les auteurs pressentis virent leur travail intégré à un colloque organisé dans l'amphithéâtre Richelieu, en Sorbonne, sur L'actualité du Génocide des Arméniens, les 16-18 avril 1998. Assis à côté d'André Kaspi dans la table-ronde de synthèse, j’insistai sur les dérives des histoires nationalistes et la nécessité de la reconnaissance de l'Autre par la Turquie contemporaine $^{7}$, un écho de l'admirable préface que Pierre Vidal-Naquet fit parâtre pour Le crime de silence. Le génocide des Arméniens ${ }^{8}$. Lorsque l'équipe pluridisciplinaire (EA 2626-CERHIC) à laquelle j'ai appartenu comme professeur à l'université de Reims de 2004 à 2008 (en la dirigeant de 2004 à 2006), organisa une série de séminaires couronnés par un colloque sur Les discours de la haine, je proposai une analyse du discours de haine contre les Juifs, dont la première expression connue est rassemblée dans le Contre Apion (I, 219-320) de Flavius Josèphe ${ }^{9}$, qui est un vrai Bréviaire de la haine au sens

6 1/1994: La société malade du moralisme; 2/1994: Etre Français?; 3/1994: Vers la société de non-communication?; 4/1994: Les juges contre la République?; 5/1995: Crises dans la sexualité? En cette année 2009 où le monde est ébranlé par une crise majeure, je relis avec intérêt le Manifeste de la revue écrit par Yves Roucaute, "Vive la politique!", Crises 1/1994, p. 5 : «L'effondrement de l'empire soviétique ne ruine pas seulement les anciennes conceptions du monde, il annonce aussi l'entrée dans une ère de crises dont nul ne semble avoir pris la démesure. Crises économiques, sociales et politiques, qui dissolvent, par atomisation et exclusion, les anciennes solidarités familiales et sociales et mettent en question, par-delà le jeu de l'État, la possibilité même du jeu politique. Crises de la subjectivité surtout, qui conduisent les individus à ne plus savoir qui ils sont, ni où ils vont, persuadés seulement qu'ils vivent dans une société bloquée dont les dirigeants sont autant de contremodèles ".

7 Bernard Legras, L'actualité du Génocide des Arméniens (Actes du colloque organisé par le Comité de Défense de la Cause Arménienne). Préface de Jack Lang, Claire Mouradian et alii éd., Paris, édipol/CDCA, 1999, p. 447-449.

8 Pierre Vidal-Naquet, "En guise de préface et par le pouvoir d'un mot...”, dans Gérard Chaliand et al. éd., Le crime de silence. Le génocide des Arméniens. Tribunal permanent des peuples, Champs-Flammarion, 1998, p. 12-13, qui renvoie à Nadine FresCO, "Les redresseurs des morts", Temps modernes, juin 1980, p. 2111-2180: "Autant dire que l'inexistence du grand massacre des Arméniens, qu’on appellera après la Seconde Guerre mondiale le génocide des Arméniens, deviendra en Turquie une vérité d'État, mieux, une vérité nationale, avec une dimension totalitaire, que tous, gouvernants, diplomates, universitaires et même professionnels de l'histoire prendront en charge. La loi de ce genre de discours s'exprime parfaitement dans le witz que Nadine Fresco a opportunément rappelé à propos du génocide des Juifs et qu'on pourrait transposer ainsi: “Il n’y a pas eu de génocide des Arméniens; ce génocide était entièrement justifié; les Arméniens se sont massacrés euxmêmes; ce sont eux qui ont massacré les Turcs".»

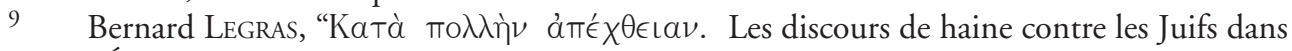
l'Égypte ptolémaïque”, dans Marc DelEPLACE éd., Les discours de la haine. Récits et figures 
où l'entendait Léon Poliakov ${ }^{10}$. L'historien de l'Égypte grecque et romaine a le devoir de s'interroger sur ce qui rendit possible cette extermination totale des Juifs, hommes, femmes, enfants, vieillards dans la province romaine d'Égypte par les troupes romaines en $115-117^{11}$.

J'attribue une importance décisive dans ma vocation d'historien à l'appartenance à une génération passionnée par le débat sur la cité. Mai 68 a été pour le lycéen de sixième un moment important. Sans être engagé, j'étais intrigué par l'ambiance d'un lycée occupé, qui vit naître les Comités d'Action Lycéens (CAL) en décembre 1967, et où flottèrent de mai à juin 1968 à la place du drapeau tricolore, les drapeaux rouge et noir ${ }^{12}$. L'atelier artisanal d'affiches recevait tous les jours ma visite. Ce fut le début d'une collection d'affiches politiques constituée grâce à l'aide de tous les militants essentiellement de gauche, d'extrême gauche et des mouvements sionistes bien implantés dans le lycée. J'ai vécu intensément ces moments que furent les grandes grèves lycéennes contre la loi Debré de février-avril 1973 sur la remise en cause des sursis militaires, les campagnes présidentielles de 1974 et 1981. La «Révolution des œillets» au Portugal, la chute des colonels grecs et le retour de la Grèce à la démocratie en 1974 (retour terni par la tragédie de l'invasion turque du Nord de Chypre), marquèrent mes années d'hypokhâgne et de khâgne. Les obsèques de Sartre, mort le 15 avril 1980, l'année où je passai l'agrégation, sont restées inoubliables. Je quittai la bibliothèque de la Sorbonne avec mauvaise conscience, me demandant si j'avais raison d'abandonner mon poste de travail quelques heures à quelques semaines des fatidiques épreuves. Je n'ai jamais regretté cette escapade au cimetière voisin du Montparnasse. C'était un adieu à un maître, un philosophe engagé dont le khâgneux que j'étais avait parcouru l'œuvre philosophique et littéraire avec avidité. La conception existentialiste de la liberté ( Nous n’avons jamais été aussi libres que sous l'Occupation") me séduisait autant que les thèses de Simone de Beauvoir sur le fait que les sexes étaient une construction culturelle.

Parmi les influences reçues, je pense que le fait d'avoir résidé, pour l'essentiel, dans des quartiers parisiens multiculturels (Château Rouge-Barbès, ouvert aux cultures $\mathrm{du}$ Maghreb et d'Afrique subsaharienne, puis le IX ${ }^{\mathrm{e}}$ arrondissement de Paris, riche de ses cultures arméniennes, grecques et juives, ashkénazes et sépharades) a aussi joué un

de la passion dans la Cité. Regards croisés, collection Histoire et Société, Villeneuve d'Asq, Presses Universitaires du Septentrion, 2009, p. 29-43.

10 Léon Poliakov, Brévaire de la haine. Le III Reich et les Juifs, Paris, Calmann-Lévy, 1951 (rééd. 1993).

11 Joseph Mélèze Modrzejewski n’a eu de cesse de s’interroger aussi sur la question. Cf. en dernier lieu sa traduction commentée de La Bible d'Alexandrie. 15.3. Troisième Livre des Maccabées, Le Cerf, 2008, en part. p. 109-113, où il revient sur l'assimilation de la communauté juive d'Égypte à un groupe de traîtres qui complote contre le souverain lagide, dans une analogie frappante avec les rebelles égyptiens.

12 Sur le mouvement lycéen au Lycée Jacques-Decour, cf. Didier LesCHI, "Les lendemains de Mai 68", Matériaux pour l'histoire de notre temps 11 (1988), p. 260-264. 
rôle dans la constitution de mes centres d'intérêts historiques. En écrivant ce texte je me suis aussi rendu compte que cet intérêt pour les rencontres et les contacts entre civilisations avait un parallèle dans mes lectures proprement littéraires sur l'Égypte moderne et contemporaine. La découverte de la Grèce moderne avait joué dans mes années adolescentes un rôle important dans ma vocation d'helléniste. La découverte des sites de Memphis et de Saqqara joua le même rôle lorsque le maître de conférences que j'étais alors détermina le sujet du mémoire inédit d'HDR, une enquête sur les reclus grecs $\mathrm{du}$ Sarapieion de Memphis au II siècle av. n.è. C'est en découvrant les statues grecques de l'Hémicycle des poètes et des philosophes, encore visibles in situ sous leur auvent de béton, puis le site intégralement recouvert par les sables du sanctuaire de Sarapis, que l'idée du travail intellectuel de recherche s'est fécondée à la vue de ce paysage de désert âpre et exaltant. Les auteurs que j'ai lus et relus étaient autant ces Grecs d'Égypte que sont le poète alexandrin Constantin Cavafy ou le romancier cairote Stratis Tsirkas, que les Juifs d'Égypte Jacques Hassoun ou Robert Solé, les Égyptiens Alaa El Aswany, Sonallah Ibrahim ou Naguib Mahfouz, ou les étrangers de passage comme Edward M. Forster ou Lawrence Durell. De Justine, je retiens autant les passages consacrés à Alexandrie, "capitale de la Mémoire», à l'antique Alexandrie "peuplant un stupéfiant espace-temps historique comme des personnages vivants", que la description d'une Égypte intemporelle avec l'évocation de la partie de chasse sur le lac Maréotis dont à l'automne «les eaux perdaient leurs scintillements d'or et tournaient au gris la pigmentation de l'hiver ${ }^{13}$ ».

\section{La construction d'une problématique de recherche: bilan en 2009}

\section{Le droit grec et hellénistique}

Parmi les questions essentielles que pose cette problématique de la Chaîne des savoirs, il faut citer en premier lieu la position que j'adopte dans cette chaîne de chercheurs qui ont constitué une discipline, la papyrologie grecque. J'ai toujours considéré que l'historien devait interroger l'ensemble des documents à sa disposition, et que la division, qui existe depuis le début du $\mathrm{Xx}^{\mathrm{e}}$ siècle, entre papyrologie historique, juridique et littéraire, ne devait s'imposer au papyrologue que comme des catégories qui doivent être transcendées. Ceci au même titre que l'indispensable recours aux autres sources concernant l'Égypte grecque et romaine: épigraphie, sources littéraires, archéologiques, iconographiques et numismatiques. L'histoire est totale sinon elle n'existe pas. J'ai montré ailleurs comment était né ce domaine de recherche qui est une branche du droit

13 Lawrence Durell, Le quatuor d'Alexandrie. Justine, éd. Le Livre de poche (trad. Roger Giroux), p.319, p.298-305, p.355. 
grec, la Juristische Gräzistik, qui s'est dégagée lentement de l'emprise du droit romain, avec Ludwig Mitteis (1859-1921), Raphael Taubenschlag (1881-1958), Hans-Julius Wolff (1902-1983) ${ }^{14}$, et Joseph Mélèze Modrzejewki (né en 1930). Ma formation est celle d'un agrégé d'histoire, alors que les savants qui ont construit cette discipline sont issus des facultés de droit, en particulier de droit romain. J'ai reçu ma formation à la papyrologie documentaire grecque d'abord dans le cadre de l'enseignement de Joseph Mélèze comme professeur d'histoire grecque à l'université Paris 1 Panthéon-Sorbonne, puis à l'EPHE $\mathrm{IV}^{\mathrm{e}}$ section au sein du séminaire «Papyrologie et histoire des droits de l'antiquité» conduit de 1972 à 2007. Son enseignement, appuyé par la Chronique papyrologique qu'il assure dans la Revue historique de droit français et étranger, a assuré le rayonnement de la papyrologie juridique auprès de générations de collègues, d'étudiants et d'auditeurs. Il fut le lieu où se rencontraient un grand nombre des collègues et amis papyrologues et historiens. La méthode suivie par Joseph Mélèze était fondée sur la constitution de dossiers qui s'enrichissaient au fil des séminaires. Les documents lus, de préférence par les auditeurs grecs qui restituaient la langue en dêmotiki, étaient scrupuleusement traduits, mot à mot, pour permettre le commentaire juridico-historique. Les auditeurs faisaient peu de conférences à la différence d'autres séminaires de l'EPHE. Ce séminaire baignait dans une ambiance internationale, voire cosmopolite, qui m’a durablement marqué. Elle venait tant de la personnalité de Joseph Mélèze, Juif venu de Pologne, qui maniait avec aisance les langues européennes, et qui n’hésitait pas à montrer, devant des auditeurs ignorants, les richesses de la langue polonaise, et ne manquait jamais de souligner les parallèles linguistiques, mais aussi par une présence de collègues étrangers qui fut permanente jusqu'au dernier séminaire le 28 mai 2007: les Grecs Sophie Adam, Julie Vélissaropoulos, Andreas Helmis et Stavros Pérentidis; les Italiens Silvia Bussi, Eva Cantarella, Daniele Foraboschi et Alberto Maffi; les Polonais Dariusz Dlugosz et Adam Lukaszewicz; les Israéliens Lucien Poznanski et Yaakov Stern; ceux venus d'Allemagne, de Belgique ou des États-Unis, Schafik Allam, Michael Gagarin, Hans Hauben et Jean A. Straus ${ }^{15}$. Cet ultime séminaire consacré au procès politique de Jésus, condamné comme agitateur pour lèse-majesté, ne fut pas empreint d'une tristesse particulière. C'est seulement à l'automne 2007 que le maître annonça que ce rendez-vous immuable depuis 1977 , le lundi à $11 \mathrm{~h}$, dans la Salle d'égyptologie de l'EpHe, avait pris fin. Une page se tournait. Cela signifiait aussi que les agapes qui suivaient le séminaire ne se tiendraient plus. Le chemin de l'Acropole, excellent restaurant grec (constantinopolitain) de la rue de l'École de médecine, devait désormais être pris dans d'autres occasions.

14 Biographie de Hans-Julius Wolff par son fils Joseph Georg Wolff dans Hans-Julius WolfF, Vorlesungen über Juristische Papyruskunde, Berlin, Duncker \& Humblot, 1998, p. 9-17.

15 Cf. l'Annuaire de l'EPHE-IV' section qui recense scrupuleusement l'identité des auditeurs et auditrices. 
La question se pose de la distance que j’ai développée par rapport à un maître avec lequel la discussion scientifique ne s'est pas interrompue avec la fin de son séminaire. J'ai sans doute accordé plus de place à la violence dans l'analyse de la société de l'Égypte hellénistique ${ }^{16}$, à la problématique du gender ${ }^{17}$, et à la documentation démotique ${ }^{18}$. Il s'agissait là d'une question de génération, non d'un désir de tuer le Père. J'entrais dans cette discipline en développant ma propre idiosyncrasie. Le sentiment de continuité est fondateur.

\section{La paideia grecque au double sens d'éducation et de culture}

Cette problématique fut première. Elle s'exprima dans un mémoire de maitrise consacré à l'institution fondamentalement grecque qu'est l'éphébie dans l'Égypte grecque et romaine. À l'origine je pensais traiter exclusivement le thème des Fêtes éphébiques. Je me souviens de ce rendez-vous au café «l'Écritoire», place de la Sorbonne, où François Aron et Joseph Mélèze Modrzejewski, unis par une même analyse sur mon projet (j'avais 21 ans), me convainquirent qu'il fallait élargir l'étude en intégrant le facteur institutionnel et juridique. La jeunesse n'était pas qu'une époque de fêtes. J'intégrais ce jour - sans le savoir - la cohorte des chercheurs qui, en ajoutant les études juridiques à la thématique de leur orientation scientifique, travaillaient en fait sur l'homme hellénistique d'Égypte dans toutes ses facettes.

Le tournant vers l'Égypte égyptienne peut être daté de la préparation de la communication que je présentai en 1998 lors du colloque organisé à Toulouse par Jean-Marie Pailler et Pascal Payen "Que reste-t-il de l'éducation classique? Relire "le Marrou" Histoire de l'éducation dans l'Antiquité». J'y prenais en compte de manière déterminante le contexte égyptien, ce qui n'avait pas été le cas dans un livre consa-

16 Je rejoins en cela Jean-Marie BerTrand, "Réponse à Joseph Mélèze Modrzejewski", Symposion 2007 (Durham, 2-6 septembre 2007), E. Harris et G. Thür éd., 2008, p. 247.

17 Joseph Mélèze Modrzejewski accordait une vraie place à l'histoire des femmes en Égypte. Le séminaire de l'EPHE, la première année où j’y fus auditeur, en 1977/78, était consacré au mariage et à la condition de la femme mariée en Égypte à l'époque ptolémaïque et sous la domination romaine. C'est là que j'ai noué des amitiés enrichissantes avec Jacqueline Christien, historienne de Sparte, et Marie-Henriette Quet, historienne de la seconde Sophistique et spécialiste des mosaïques gréco-romaines.

18 Le parcours d'historien de Joseph Mélèze Modrzejewski l'a poussé, au fil des années, à privilégier l'étude du judaïsme hellénistique en Égypte, d'où l'approfondissement continu de sa connaissance de l'hébreu biblique et moderne, alors que je m'orientais vers une connaissance toujours plus intime de l'Égypte «égyptienne» marquée par le début de l'apprentissage de l'égyptien démotique avec Ghislaine Widmer, puis avec Michel Chauveau au sein de son séminaire de démotique à l'EPHE $\mathrm{IV}^{\mathrm{e}}$ section. Mais son séminaire était suivi assidûment par l'égyptologue Schafik Allam, venu de Tübingen, et les travaux des historiens du droit pharaonique, en particulier de Bernadette Menu, étaient largement utilisés. 
cré à la paideia grecque dont la première édition parut précisément en $1998^{19}$. Ma communication insérée dans une troisième partie «Relectures régionales; l'universel et le particulier» s'intitulait "Entre grécité et égyptianité: la fonction culturelle de l'éducation grecque dans l'Égypte hellénistique ${ }^{20}$.» Pauline Schmitt Pantel, qui suscita mon invitation à ce colloque, a conduit - sans en mesurer sans doute toutes les conséquences - à une nouvelle orientation majeure de la recherche. C'était aussi le moment où j'achevais la réécriture de ma thèse, qui devait être publiée l'année suivante, en intégrant plus que je l'avais fait dans le manuscrit initial les sources démotiques ${ }^{21}$. C'est aussi dans ce nouveau contexte intellectuel qu'est née cette synthèse issue d'un cours en Sorbonne, Lire en Égypte, d'Alexandre à l'slam, fondée sur la documentation grecque et égyptienne ${ }^{22}$. Le compte rendu de Willy Clarysse me convainquit de l'intérêt de l'entreprise, qui me faisait comprendre que j'avais échappé - au moins en partie - aux pièges de l'hellénocentrisme ${ }^{23}$. Cette nouvelle orientation fut marquée dans ma géographie des bibliothèques de recherche par une fréquentation de plus en plus assidue de la Bibliothèque d'égyptologie "Champollion" au Collège de France.

Dans ce domaine, je trouvai une voie propre où les travaux de Pierre VidalNaquet sur les classes d'âges (Le chasseur noir), les recherches sur les jeunes gens et les jeunes filles, issues des chercheurs plus proches de ma génération (Pierre Brulé, Andrej Chankowski ou le médiéviste Jean-Claude Schmitt), et les travaux sur l'identité grecque en Égypte de Joseph Mélèze Modrzejewski étaient autant de nourritures spirituelles.

\section{Les transferts culturels}

L'intérêt pour ce concept est né au sein du séminaire de recherche animé depuis 2002 au sein de l'équipe Phéacie avec Jean-Christophe Couvenhes. Je dois à Pauline Schmitt Pantel, directrice de Phéacie/Paris 1, d'avoir bénéficié du don le plus précieux, l'exercice de la liberté intellectuelle. Les principes qu'elle mit en ouvre, avec les fondateurs de Phéacie issus de Paris 7, ont posé un cadre intellectuel de recherche, dont les bénéfices ont continué à me stimuler durant mes années rémoises (2004-2008). Le fonctionnement collégial et démocratique de Phéacie, une équipe d'accueil, offrait un cadre

19 Bernard Legras, Éducation et culture dans le monde grec (VIII siècle-Ire siècle av. J.-C.), Paris, Sedes, collection Campus, 1998; $2^{\mathrm{e} e ́ d .: ~ E ́ d u c a t i o n ~ e t ~ c u l t u r e ~ d a n s ~ l e ~ m o n d e ~ g r e c ~(V I I I ~ s i e ̀ c l e ~}$ av. J.-C.-IV siècle ap.J.-C.), Paris, Armand Colin, collection Cursus, 2002. Traduction en grec: éditions Kritiki, Athènes, 2005.

20 Publié en 2004 aux Presses universitaires du Mirail.

21 Bernard LEgras, Néotês. Recherches sur les jeunes Grecs dans l'Égypte ptolémä̈que et romaine, Genève, Droz, Paris, collection Hautes Études du Monde gréco-romain, EPHE, IV Section, 1999.

Bernard Legras, Lire en Égypte d'Alexandre à l'Islam, Paris, Picard, collection Antiqua, 2002.

23 Willy Clarysse, Paedagogica Historica 40 (2004), p.825-828. 
épanouissant, où l'harmonie et la solidarité qui y régnaient ont créé un groupe de chercheurs unis par le sentiment de participer à une aventure intellectuelle exceptionnelle. Deux colloques furent organisés. Une première Rencontre organisée en Sorbonne, en février 2004 sur les Transferts culturels et politique dans le monde hellénistique a permis de valider l'hypothèse de sa fécondité pour les historiens de l'Antiquitée ${ }^{24}$. Elle s'appuyait sur un concept théorisé dans un cadre franco-allemand par des civilisationnistes, Michel Espagne (ENS) et Michael Werner (EHESS-CRIA), qui fut l'objet d'un Séminaire organisé par l'École doctorale d'histoire de l'université Paris 1 PanthéonSorbonne ${ }^{25}$. Ce colloque de six communicants restait cependant très hellénocentré. Les deuxièmes Rencontres, à Reims, en mai 2008, ont eu pour ambition de s'attacher à la sphère des droits de l'antiquité, grecs et «barbares» dans le cadre de leurs réceptions réciproques. Il s'agissait de déterminer comment ces droits se transforment du fait d'une évolution interne ${ }^{26}$. La problématique envisagée imposait des échanges et des débats entre spécialistes venus d'horizons différents, historiens, juristes, anthropologues, hellénistes, romanistes, démotisants, sémitisants, orientalistes, dont les regards sur le monde grec et hellénistique sont complémentaires. Les deux enjeux principaux de ce Congrès étaient, d'une part, de revenir sur les dynamiques juridiques internes au monde grec et, d'autre part, de mesurer les influences réciproques des droits des mondes grecs et des mondes «barbares». L'armure était fendue. L'helléniste, qui s'était ouvert aux contacts scientifiques avec les démotisants, Michel Chauveau à Paris, et Willy Clarysse à Louvain, s'ouvrait sans retenue intellectuelle aux autres cultures du monde hellénistique. C'est au contact de ces chercheurs, mais aussi d'autres comme Roger Bagnall ou Françoise Dunand, que je me suis interrogé sur la nature de la société ptolémaïque. Alors que je la définissais encore en 1995 comme une société coloniale ${ }^{27}$, le terme de société multiculturelle s'est imposé dès 1998 à partir du colloque de Toulouse consacré à Henri $\mathrm{Marrou}^{28}$. La question de la place de l'univers religieux égyptien s'est posée de manière centrale lors de l'écriture de mon livre sur les reclus grecs du Sarapieion ${ }^{29}$. Leur réclusion est en effet interprétée traditionnellement - je schématise une question complexe - soit comme l'expression de la séduction exercée par la vie religieuse dans

24 Jean-Christophe CouvenHES et Bernard Legras éd., Transferts culturels et politique dans le monde hellénistique (Paris, 7 février 2004), Publications de la Sorbonne, 2006.

25 Hypothèses, Publications de la Sorbonne, 2003, p.149-218, Béatrice Joyeux éd.

26 Bernard Legras (éd.), Transferts culturels et droits dans le monde grec et hellénistique, (Reims, 14-17 mai 2008), Paris, Publications de la Sorbonne (à paraître).

27 Bernard Legras, "Économie et société à l'époque hellénistique", dans Michel Kaplan éd., Le monde grec, Bréal, Grand Amphi, 1995, p.311-346. J'ai modifié ce point de vue dans la seconde édition revue et corrigée à paraitre fin 2009.

28 Bernard Legras, L'Égypte grecque et romaine, Paris, Armand Colin, 2004 (réimpr. 2009), p. 162-184.

29 Bernard Legras, Les reclus grecs du Sarapieion de Memphis. Une enquête sur l'hellénisme égyptien, Louvain (Leuven), Studia hellenistica, Peeters (à paraître). 
le sanctuaire, soit comme le résultat d'un destin "laïc» fondé sur la volonté de trouver refuge dans un espace protecteur. Jean-Marie Bertrand savait - comme personne - me pousser dans mes derniers retranchements intellectuels. Je lui dois un exemple de rigueur et de bienveillance intellectuelles dans la direction d'une HDR. Les parallèles qu'il me suggéra vers d'autres espaces du monde hellénistique contribuèrent considérablement à une mise en perspective plus globale de ma problématique tournée vers l'hellénisme égyptien. Et je dois à Willy Clarysse de m'avoir mieux fait percevoir certaines données proprement égyptiennes du dossier papyrologique des reclus du Sarapieion de Memphis. La Mischforschung appliquée à l'Égypte grecque et romaine nécessite une étroite collaboration entre hellénistes et démotisants. Ces discussions lovaniennes ont contribué de manière décisive à la conception que j'ai désormais de mon métier d'historien. La coordination d'une publication destinée à un public cultivé sur les «Dieux de l'Égypte" a confirmé, d'une certaine manière, mon entrée dans l'étude de la conception du monde vu par le clergé égyptien ${ }^{30}$.

En affirmant la place des transferts culturels dans la recherche historique, j'ai prolongé et renouvelé des thèmes chers à Joseph Mélèze Modrzejewski qui a posé sans relâche la question de la réception des lois, et dont la volonté comparatiste a été constante. Le séminaire commun des équipes Gernet-Glotz-Phéacie du lundi 17 h-19 h que j'ai animé en 2007/2008 avec Stella Georgoudi et Jean-Louis Ferrary sur le thème de "La normativité", aura été à ce titre un lieu important d'échanges et d'enrichissement ${ }^{31}$. Ce concept élaboré au milieu des années 1980 s'affirme dans la continuité de ce "passeur entre les cultures» qu'est un maître respecté devenu, au fil des ans, un ami avec lequel les liens sont aujourd'hui ceux de la philia.

Bernard LEgras

Université Paris 1 Panthéon-Sorbonnel

Équipe Phéacie (EA 3563)

4, rue de Chantilly

F- 75009 Paris

bernard.legras@univ-paris1.fr

30 Bernard Legras, "Un panthéon foisonnant", dans Les dieux de l'Égypte ancienne, Textes et Documents pour la Classe (TDC), coord. B. Legras, Paris, SCÉRÉN/CNDP, n 950, 15 février 2008, p. 6-13.

31 Cf. ma communication du 5 février 2007, "Le droit familial dans le statut augustéen de l'Égypte: de nouvelles normes?" (à paraître dans Métis). Ce séminaire m’aura aussi ouvert vers les programmes de recherches des UMR Gernet et Glotz dans la perspective de la fusion en 2010 des équipes Gernet-Glotz-Phéacie au sein d'une UMR, "Histoire et anthropologie des mondes antiques". 